\title{
La périlleuse transition démocratique au Pakistan
}

\section{Gilles Boquérat}

\section{(2) OpenEdition \\ Journals}

\section{Édition électronique}

URL : http://journals.openedition.org/echogeo/5883

DOI : 10.4000/echogeo.5883

ISSN : 1963-1197

\section{Éditeur}

Pôle de recherche pour l'organisation et la diffusion de l'information géographique (CNRS UMR 8586)

\section{Référence électronique}

Gilles Boquérat, «La périlleuse transition démocratique au Pakistan », EchoGéo [En ligne], Sur le Vif, mis en ligne le 23 juin 2008, consulté le 19 avril 2019. URL : http://journals.openedition.org/ echogeo/5883 ; DOl : 10.4000/echogeo.5883

Ce document a été généré automatiquement le 19 avril 2019.

Licence Creative Commons

EchoGéo est mis à disposition selon les termes de la licence Creative Commons Attribution - Pas d'Utilisation Commerciale - Pas de Modification 4.0 International 


\title{
La périlleuse transition démocratique au Pakistan
}

\author{
Gilles Boquérat
}

1 A ceux qui désespéraient que le Pakistan soit condamné à être seulement dirigé par Allah, les Américains et l'Armée, les élections du 18 février 2008 ont montré que l'Awam (le peuple) avait encore son mot à dire en infligeant un cuisant revers au président Musharraf. Les résultats ont démontré une nouvelle fois que la longanimité du peuple pakistanais envers ses dirigeants militaires n'était pas indéfectible et que le maintien au pouvoir de ces derniers devait souvent moins à leur popularité qu'à des manipulations électorales. Ils donnèrent largement raison à Benazir Bhutto qui avait appelé à ne pas boycotter les élections malgré les incertitudes pesant sur leur bon déroulement. Nul doute que son assassinat le 27 décembre 2007 contribua au revers subi par le pouvoir en place, moins d'ailleurs en termes de gains électoraux pour son parti qu'en contribuant à renforcer la défiance vis-à-vis de Musharraf et en rendant plus difficile les fraudes. Avec un verdict balayant toute équivoque quant à la volonté des électeurs de tourner le dos au régime autocratique, encore fallait-il que les chefs de la nouvelle majorité, Asif Ali Zardari et Nawaz Sharif, s'entendent pour s'attaquer d'urgence à une situation de crise multiforme. Beaucoup voulurent croire à la réconciliation des adversaires d'hier lorsque le 9 mars 2008, un an jour pour jour après le limogeage du chef de la Cour Suprême qui signala le début des ennuis pour Musharraf, ils adoptèrent la déclaration de Murree. Elle prévoyait un inédit partage du pouvoir entre le Pakistan Peoples Pakistan (PPP) et la Pakistan Muslim League-Nawaz (PML-N) et l'adoption d'une résolution parlementaire dans les trente jours suivant la formation d'un gouvernement de coalition permettant le rétablissement dans leurs fonctions des juges démis lors de l'imposition du peu constitutionnel état d'urgence le 3 novembre 2007. L'incapacité du gouvernement conduit par le PPP à respecter l'échéance fixée est le reflet de la difficulté à bâtir de nouveaux équilibres institutionnels. L'impasse sur la question du rétablissement de tous les juges, dont la PML-N avait fait son cheval de bataille électoral, obligeant même ses candidats à une prestation de serment en ce sens, a conduit ce parti le 12 mai à retirer ses ministres du gouvernement. Simple incident de parcours ou rupture bientôt consommée entre les vainqueurs des élections de février, le Pakistan n'en finit pas d'être à la recherche d'une 
stabilité politique cruellement nécessaire pour s'attaquer aux pénuries et enrayer le sentiment croissant de frustrations d'une population qui s'était félicitée en février de retrouver une respectabilité démocratique aux yeux de la communauté internationale.

\section{La flèche, le tigre et la bicyclette}

2 Le premier enseignement des élections du 18 février est leur relative régularité, Certes le gouvernement intérimaire n'était pas vraiment neutre et l'impartialité de la commission électorale fut suspecte, mais s'il y eut des fraudes le jour des élections (notamment des taux de participation parfois surprenants), les exactions restèrent suffisamment limitées pour ne pas remettre en cause l'orientation globale du scrutin. Sûrs de surfer sur une vague anti-Musharraf, Asif Zardari et Nawaz Sharif avaient prévenu que si leur parti réciproque n'occupait pas les deux premières positions à l'issue du scrutin, celui-ci serait considéré comme truqué. Il n'y eut donc rien de comparable aux précédentes élections générales de 2002 dont le général Ehtasham Zameer, alors chef de la cellule politique de l'Inter-Services Intelligence (ISI), reconnut qu'elles avaient été manipulées pour faire gagner - difficilement pourtant - le parti du président, la PML-Quaid-i-Azam (PML-Q)1. Cette fois-ci, le successeur de Pervez Musharraf à la tête des forces armées, le général Ashfaq Parvez Kayani, avait annoncé que les agences de renseignements militaires - MI (Military Intelligence) et ISI - n'interféreraient pas dans le processus électoral le jour du vote.

3 Les irrégularités constatées ont plutôt porté sur la campagne électorale au cours de laquelle la PML-Q a bénéficié d'un traitement de faveur des autorités locales. Au Pendjab, dans 33 des 35 districts, les nazims (chefs de district) élus en 2005 étaient des proches de la PML-Q et eurent peu de scrupules à soutenir le candidat de ce parti, qui était d'ailleurs souvent un membre de leur famille. Si l'électorat a changé avec l'urbanisation, l'éducation et la dissémination de moyens de communication moderne, et a rajeuni (environ $55 \%$ de la population a moins de 25 ans), il n'en est pas de même de la composition sociale de la classe politique. Ce sont encore les grands propriétaires terriens qui dominent quand il s'est agi de recevoir l'investiture du PPP et des deux PML, suivis par les hommes d'affaires ${ }^{2}$.La PML-Q eut aussi les ressources et l'influence nécessaires pour battre la campagne sans entraves et s'arroger une incomparable visibilité dans les médias. A l'inverse, le PPP, qui avait décrété quarante jours de deuil après l'assassinat de Benazir Bhutto, n'eut guère l'occasion d'organiser de grands rassemblements publics.

4 Sur la base des symboles électoraux, la bicyclette (la PML-Q) a été nettement distancée par le tigre (la PML-Nawaz) et la flèche (le PPP). Les appels au boycott furent limités à des partis relativement secondaires (le Jamaat-e Islami, le Tehrik-i-Insaf, des partis autonomistes comme le Pakhtoonkhwa Milli Awami Party (PMAP). Le taux de participation officiel $(45 \%)$, le second plus haut jamais enregistré lors d'élections générales, témoigna, d'une mobilisation relativement importante, même s'il exista de larges variations régionales. Après bien des tergiversations et finalement aiguillonné par le PPP, le parti de Nawaz Sharif, avait décidé de concourir. Bien lui en prit puisqu'il émergea comme le premier vainqueur des élections, passant de 18 députés en 2002 à 90 en 2008, prenant même 14 sièges au PPP sans en céder aucun à ce parti ${ }^{3}$. Toutefois, la PML-N reste fondamentalement un parti dont les forces sont concentrées au Pendjab, puisque plus de $90 \%$ de l'ensemble de ses députés vient de cette province où le parti remporta $40 \%$ des sièges en jeu ${ }^{4}$. Cette prééminence de la PML-N est particulièrement 
nette dans le nord et le centre de la province, qui comprennent les grands centres urbains, abandonnant comme d'habitude le sud, plus rural, au PPP. La seule incursion de la PML-N hors du Pendjab fut dans leur fief traditionnel des districts contigus de la province de la frontière du nord-ouest (NWFP) de langue hindko (Abbotabad, Haripur, Mansehra). La PML-N a su plus que les autres partis mobiliser au Pendjab les abstentionnistes de 2002 et la classe moyenne qui a été particulièrement sensible au mouvement des avocats dirigé.

5 Le bonheur de la PML-N a inévitablement fait le malheur de la PML-Q qui chuta de 118 à 51 élus. Facteur aggravant, nombre de figures prééminentes du parti furent battues, à commencer par son président, Chaudhry Shujaat Hussain. Au total, ce sont 23 ministres de Musharraf qui furent défaits ${ }^{5} . I$ apparaît que la PML-Q a proportionnellement mieux réussi dans les régions les plus déshéritées mais qui sont aussi les plus aisément placées sous l'influence d'un potentat de province en phase avec le pouvoir central ${ }^{6}$. La nette poussée de la PML-N a relativement freiné la progression escomptée du PPP. La vague de sympathie attendue après l'assassinat de Benazir Bhutto s'est transformée en vaguelette. La PML-N a probablement été récompensée pour son inflexible opposition à Musharraf là où le PPP put apparaitre comme enclin aux compromissions. Benazir Bhutto avait négocié avec Musharraf, et sous patronage américain, les termes de son retour d'exil, ce qui s'était traduit, la veille de la controversée réélection de Musharraf, par l'exonération des accusations de corruption (l'ordonnance de réconciliation nationale du 5 octobre 2007) portées à son encontre et envers un certain nombre d'hommes politiques, dont son mari, Asif Ali Zardari ${ }^{7}$.

6 Au Sind, la fracture socio-politique entre d'une part la trilogie PPP/Sindhis/ campagnes et d'autre part celle Muttahida Qaumi Movement (MQM)/ Mohajirs/villes s'est confirmée. En l'absence notamment du JI, le MQM consolida son emprise sur le Sind urbain, augmentant même son électorat ( $85 \%$ des sièges à Karachi et $40 \%$ à Hyderabad) et il fut le seul parti à battre son record de sièges remportés lors d'élections générales. Le Sind reste toutefois la terre de prédilection du PPP. Il a significativement accru son audience en termes de votes même si le nombre de députés élus n'a que marginalement augmenté par rapport à $2002(+2$, soit 29 sièges généraux sur 58$)$.

7 Ces élections ont aussi été marquées par un net recul de la composante politico-religieuse représentée essentiellement cette fois-ci par le JUI-F (Jamiat Ulema-i-Islam-Fazal). L'Awami National Party (ANP), parti pachtoune qui se veut progressiste et laïcisant, a réémergé comme la première force politique dans la NWFP, suivi de près par le PPP. Il a remporté 10 sièges de députes, notamment dans les districts de la vallée de Peshawar (6 sièges). En 2002, à la faveur de l'intervention américaine en Afghanistan qui permit de jouer sur le registre de l'islam en danger et avec un coup de pouce des services de renseignements, le MMA (Muttahida Majlis-i-Amal), une alliance de partis religieux chapeautée par le JUI-F et le JI, avait obtenu presque la moitié des suffrages exprimés et raflé presque la totalité des sièges (29 sur 35$)$. Il y a plusieurs explications à ce recul (seulement trois sièges remportés, et 10 sièges généraux sur 76 au niveau de l'assemblée provinciale): l'absence d'amélioration du niveau de vie alors que les actes de violence sont en nette augmentation, le boycott du JI, la complaisance affichée du chef du JUI-F, Maulana Fazlur Rehman, à l'encontre de Musharraf ${ }^{8}$. Le MMA fut aussi accusé par l'électorat le plus religieux de ne pas en faire assez pour imposer la sharia (loi islamique) ou, à l'inverse, de manifester une réticence à s'attaquer de front à la militance islamiste. 
Le JUI-F a été débordé par des éléments plus extrémistes qui échappent à son contrôle avec la montée en puissance de talibans locaux.

8 Au Baloutchistan, le MMA a aussi reculé, alors qu'en 2002 il avait bénéficié d'un appui du gouvernement aux groupes islamistes pachtounes afin de marginaliser l'influence des nationalistes baloutches. Bien que les opérations militaires menées depuis 2005 aient exacerbé les tensions et les mécontentements, c'est au Baloutchistan que la PML-Q réalisa, relativement parlant, sa meilleure performance avec 5 élus nationaux (sur 17) et 20 sièges de députés provinciaux (sur 63). Un paradoxe qui peut s'expliquer par un fort taux d'abstention et des personnalités élues plus du fait de leur statut social et de leur proximité des cercles d'influence que pour leur engagement politique (tous les partis représentés à l'assemblée provinciale ont décidé d'apporter leur soutien au gouvernement dirigé par le PPP) ${ }^{9}$. Le boycott des partis nationalistes (pachtoune dans le cas du PMAP, et baloutches pour le National Party, le Balochistan National Party (BNP), et le Jamhoori Watan Party dont le leader, Akbar Bugti, avait été tué dans une attaque de l'armée en août 2006) a surtout bénéficié au PPP qui réalisa ainsi sa meilleure performance dans la province ( 5 députés nationaux).

S'il n'y a pas eu de raz-de-marée du PPP, ce dernier reste cependant, le premier parti du pays en termes de députés et en termes de suffrages exprimés (31\%). C'est aussi le seul parti a pouvoir revendiquer une présence significative dans toutes les provinces avec la moitié des sièges au Sind, un tiers au Pendjab, et $30 \%$ des sièges au NWFP et au Baloutchistan. En conséquence, le PPP est à la tête de gouvernements de coalition non seulement à Islamabad mais aussi dans deux des quatre gouvernements provinciaux (Sind et Baloutchistan). A ce titre, le PPP est certainement plus à l'écoute des provinces que ne peut l'être la PML-N. La décision récente d'abandonner le projet de barrage de Kalabagh décrié dans la NWFP et au Sind participe de cette sensibilisation. Même si la PML-Q a aussi réussi à faire élire des députés sur une bonne partie du territoire, c'est en bien moins grand nombre et surtout le Pendjab compte pour $70 \%$ des sièges obtenus. Au regard de ces élections, le PPP, et dans une moindre mesure la PML-Q, sont les deux seuls partis à pouvoir prétendre à une représentation dans tous les groupes ethniques et linguistiques. La PML-N émerge d'abord comme le parti de la petite bourgeoisie urbaine du Pendjab. L'ANP et le MMA restent des partis dont l'audience est essentiellement pachtoune avec une différence tenant au fait que l'électorat du MMA est avant tout rural et à faible revenu. Quant au MQM, malgré des tentatives d'élargir son électorat au-delà du pré carré du Sind urbain et la rhétorique sur la défense des milieux défavorisés, il demeure le parti des mohajirs (les immigrés et leurs descendants venus d'Inde à la Partition) relativement aisés. Cette identification à un groupe bien défini lui permet d'avoir un poids politique disproportionné. Seul l'électorat du PPP suit finalement assez fidèlement la répartition nationale entre milieux rural, péri-urbain et urbain et ces électeurs appartiennent dans une égale proportion à toutes les classes de revenus.

\section{Une configuration politique nationale et provinciale en devenir}

10 Face au rejet de Musharraf et de son parti et à la bonne performance de la PML-N, une alliance entre cette dernière et le premier parti du pays, le PPP, s'imposa puisque tel était le verdict populaire. Plate-forme d'action gouvernementale, la charte de la démocratie signée en mai 2006 à Londres par Benazir Bhutto et Nawaz Sharif pouvait constituer le 
socle de cette coopération. Des doutes subsistaient néanmoins sur la viabilité d'une telle alliance, le PPP était manifestement plus réservé sur le rétablissement inconditionnel des juges démis et certainement moins belliqueux que la PML-N en ce qui concerne le départ de Musharraf, voire même une comparution devant les tribunaux à laquelle les dirigeants militaires ont toujours échappé.

11 La PML-N, après un refus initial de partager le pouvoir avec un président jugé illégitime, se laissa donc convaincre, avec la déclaration de Murree, de participer à un gouvernement de coalition, comme il l'avait été précédemment de participer aux élections ${ }^{10}$. Le parti obtint neuf portefeuilles ministériels au niveau fédéral dont ceux des finances et de l'éducation (onze ministères sont allés au PPP, deux au parti de la ligue Awami, un au JUI-F et un à un député indépendant des FATA). Il était notamment entendu en contrepartie de cette participation au gouvernement que la PML-N aurait la haute main sur le Pendjab où Shahbaz Sharif, le frère de Nawaz, a repris début juin les fonctions de ministre en chef qu'il occupa de 1997 à 1999.

12 Si Youssuf Raza Gilani fut nommé premier ministre le 22 mars, le pouvoir réel au sein $d u$ PPP est entre les mains de Asif Ali Zardari, officiellement co-président du parti, avec son fils de 19 ans Bilawal Zardari-Bhutto. C'est d'ailleurs Zardari qui préféra Gilani, jugé moins menaçant, à un autre makhdoom(littéralement: celui qui doit être servi, le titre désigne le descendant d'un saint), le sindhi Amin Fahim. Les antécédents de Gilani illustrent certains traits récurrents d'un parcours politique au Pakistan : un engagement public familial sur plusieurs générations, des affiliations politiques fluctuantes, et le passage par la case prison. Il est l'héritier d'une famille politiquement et religieusement influente de Multan, grande ville du centre du Pendjab notamment connue pour ces mausolées de saints soufis. Gilani entra en politique en adhérant à la Ligue Musulmane et en servant comme ministre du gouvernement Junejo de 1985 à 1988 lorsque Zia ul-Haq était le président du pays. Il rejoignit ensuite les rangs du PPP pour lequel il remporta un siège en 1988, ainsi qu'en 1990 et en 1993, année durant laquelle il fut nommé président de l'assemblée nationale. Condamné pour abus de pouvoir en 2001, il refusa de négocier sa libération contre une allégeance au régime de Musharraf et passa finalement près de cinq années derrière les barreaux.

13 Autre personne à avoir été incarcéré - onze ans au total sous l'inculpation de chantage et de corruption -, Asif Ali Zardari a lissé son image pour apparaitre comme l'homme soucieux de réconciliation pour une démocratisation en douceur. Toute réforme en profondeur des institutions passe par un amendement à la Constitution et requiertune majorité des deux-tiers dans chacune des deux chambres. Un seuil susceptible d'être atteint à l'Assemblée nationale, mais plus difficilement accessible au Sénat où Musharraf peut toujours compter sur une majorité (57 sénateurs) qui devrait logiquement disparaître lors du renouvellement de la moitié des sièges en 2009.

14 L'ANP était au niveau idéologique un allié naturel, bien qu'il ait pu faire alliance électoralement avec la PML-N dans les années quatre-vingt-dix. Aux commandes du gouvernement à Peshawar est Haider Hoti, neveu de l'actuel président de l'ANP et dont la tante est l'ancienne présidente du parti, la bégum Nasim Wali Khan, qui eut aussi la distinction d'être la première femme du pays à être élue députée en 1970. Preuve encore une fois de l'importance du pedigree familial, le nouveau ministre en chef du Baloutchistan, Mohammed Aslam Raisani, du PPP, est chef de clan et fils du Nawab Ghous Bakhsh Raisani qui fut nommé gouverneur de cette province en 1972 avant de devenir ministre au niveau fédéral. 

Baloutchistan. Ce parti avait aussi l'insigne avantage de disposer de 15 sénateurs et peut être une valeur ajoutée dans l'optique de négociations ouvertes avec les militants dans les zones tribales du nord-ouest administrées par le gouvernement central (Federally Admistered Tribal Areas $=$ FATA). Le chef du JUI-F, Maulana Fazlur Rehman n'est jamais très loin des sphères du pouvoir et espère faire valoir les intérêts des éléments cléricaux - il a déjà mis en garde contre la réforme des madrassas - en marquant des points sur le JI mieux organisé mais absent du pouvoir. Moins attendues furent les ouvertures de Zardari en direction du MQM au regard des relations conflictuelles entretenues par le passé et le soutien accordé par ce dernier au régime Musharraf illustré par les violences orchestrées par le MQM au moment du déplacement de Iftikhar Chaudhry à Karachi le 12 mai 2007. Au Sind, où le PPP dispose d'une majorité absolue, la plupart de ses élus, qui n'ont pas oublié les excès commis à l'encontre de leurs militants par le MQM au cours des dernières années, étaient récalcitrants. Cependant une large place fut faite au MQM dans le gouvernement provincial, avec l'idée de neutraliser le parti régnant sur Karachi, mais aussi celui de se ménager un soutien supplémentaire au niveau national ( 25 députés). La stratégie d'ouverture de Zardari peut aussi être interprétée comme la volonté de garder la porte ouverte à des voies de recours si la coalition avec la PML-N faisait finalement long feu. régime. Musharraf n'est pas homme à lâcher prise devant des personnalités politiques qu'il tient responsables de la « démocratie factice » des années quatre-vingt-dix alors que lui-même n'hésita pas à plonger le pays dans une crise institutionnelle et constitutionnelle, en s'attaquant au pouvoir judiciaire afin de pouvoir proroger une présidence que les électeurs rejetèrent largement en février. Près des deux-tiers des 97 juges des cours supérieures avaient refusé de se soumettre à l'état d'urgence. Les indices de désaveu ne manquaient pourtant pas. Dans un sondage réalisé pour le compte du service ourdou de la $B B C$ avant les élections, il apparaissait que près des deux-tiers des personnes interrogées considéraient qu'une démission du président aiderait à la stabilité du pays et que la future assemblée nationale devrait s'employer à une réinstallation de Iftikhar Chaudhry. Seulement $16 \%$ d'entre elles étaient prêtes à suivre le gouvernement quand celui-ci clamait que le leader taliban Baitullah Mehsud et al-Qaeda étaient derrière l'assassinat de Benazir Bhutto ${ }^{11}$.

Musharraf est certes affaibli mais pas totalement isolé, pouvant encore compter, jusqu'à un certain point, sur l'establishment composé des échelons supérieurs de l'armée en collusion avec la haute fonction publique et opérant avec la complicité de personnalités politiques. Ce réseau comprend aussi les agences de renseignements dont l'ISI dirigée par Nadeem Taj, un proche du président. Sans oublier la complaisance des Etats-Unis auprès desquels Musharraf a joué et rejoué du registre de l'homme providentiel dans la lutte contre le terrorisme. Une implication plus marquée du Parlement sur les questions de sécurité nationale pourrait perturber l'action des Américains qui furent habitués à traiter directement avec Musharraf. Le soutien de l'armée n'est toutefois plus indéfectible depuis que Musharraf a quitté sa «seconde peau » même s'il demeure constitutionnellement le commandant suprême des forces armées. Elle ne soutiendrait pas actuellement une tentative de dissolution de l'assemblée nationale mais verrait aussi défavorablement une procédure de destitution. Le général Kayani a jusqu'ici donné des assurances quant au désengagement de l'armée des fonctions civiles gouvernementales et a interdit aux 
officiers en activité de rencontrer des personnalités politiques sans accord préalable. Outre consolider sa position en nommant des officiers de son choix, Kayani paraitt soucieux de reconstruire l'image de l'armée ternie par des opérations militaires guère populaires (régions tribales frontalières, Baloutchistan, mosquée rouge) alors que, fait nouveau, cette armée est la cible d'attentats-suicide depuis l'hiver 2006. Le retrait du devant de la scène ne doit toutefois pas laisser penser à un abandon de positions dominantes quant à la gestion des crises et certains aspects de politique extérieure et à un feu vert donné à un droit de regard public sur les intérêts acquis au fil du temps dans la sphère économique à travers de nombreuses fondations à but lucratif et sur la gestion de l'énorme patrimoine foncier de l'armée ${ }^{12}$. Pour la première fois, et comme la charte de la démocratie le demandait, le budget de la défense présenté au Parlement fournit un peu plus de détails sur sa répartition que l'habituelle enveloppe globale séche.

Zardari, qui n'exclut pas de viser la fonction présidentielle, doit composer avec différents acteurs dont tous ne tirent pas leur légitimité du peuple. Renversement de situation ou leçon bien apprise, le PPP, qui durant les années quatre-vingt-dix était en confrontation quasi-permanente avec l'establishment apparaît aujourd'hui comme le parti le plus disposé à faire preuve de conciliation à l'inverse de Nawaz Sharif, dont la carrière politique avait été pourtant lancée par ce même establishment.

Si Musharraf parvient à éviter une procédure de destitution pour violation de la Constitution (un vote à la majorité des deux-tiers de l'ensemble des deux chambres est nécessaire) et s'il ne se résigne pas à abandonner de lui-même la fonction présidentielle sous la multiplication des pressions, il lui sera toutefois difficile de s'opposer à une réduction significative de ses pouvoirs, à commencer par la suppression de l'article 58 (2b) qui permet au président de dissoudre l'assemblée nationale et qui fut trop souvent utilisé par le passé pour se débarrasser du premier ministre ${ }^{13}$.En ligne de mire sont aussi les pouvoirs de nomination du président, soit à sa discrétion (le chef de la Cour Suprême, le chef de la commission électorale), soit après consultation avec le premier ministre (les gouverneurs, le chef des forces armées et des différents corps d'armée) ou avec le chef de la Cour Suprême (les juges de cette cour). De telles réformes figurent dans le paquet en 80 points que le PPP fait circuler en vue de l'adoption d'un 18e amendement à la Constitution qui entérinerait le retour au système parlementaire prévu à l'origine dans la Constitution de 1973 et redonnerait l'exercice réelle de l'autorité exécutive au gouvernement. Sur ces modifications constitutionnelles et sur d'autres, comme la charge de haute trahison pour les juges qui avaliseraient à l'avenir un coup de force des militaires, il n'y a pas vraiment de dissensions entre le PPP et la PML-N. Par contre cette dernière récuse la protection accordée aux ordonnances et décisions présidentielles prises entre le 12 juillet et 15 novembre 2007, ce qui est interprété comme la volonté de Zardari de se protéger de toutes remise en cause de l'ordonnance de réconciliation nationale et offrir à Musharraf l'opportunité de se retirer sans crainte de poursuites. Surtout, il demeure la divergence cardinale sur le rétablissement des juges démis, à commencer par Iftikhar Chaudhry, ce qui amena la PML-N à s'associer à la «longue marche » des avocats qui s'est achevée le 13 juin à Islamabad par un vaste rassemblement populaire. Ni Zardari, ni le président, ni l'establishment, ont particulièrement envie de voir revenir à la tête du pouvoir judiciaire l'homme qui entendait ré-examiner la légalité de l'ordonnance de réconciliation nationale, de la réélection de Musharraf, ou encore précédemment avait questionné les agissements de l'armée au Baloutchistan. Le chef du PPP n'oublie pas de rappeler qu'avant de devenir la «conscience» de la nation, en 
refusant de se soumettre au président, Iftikhar Chaudhry avait été parmi les juges qui avaient avalisé le coup d'état d'octobre 1999. Le PPP est accusé de manœuvre dilatoire en liant d'une part le rétablissement des juges démis à longue adoption d'un $18^{\mathrm{e}}$ amendement et, d'autre part, en proposant de maintenir dans leurs fonctions ceux qui avaient prêté serment à l'ordre constitutionnel provisoire qui suivit la déclaration de l'état d'urgence. D'ailleurs, ans le cadre du budget 2008-2009 adopté le 22 juin avec le soutien du PML-N, la loi de finances porte de 16 à 29 le nombre de juges de la Cour Suprême. Un accroissement qui permet de miser sur des discordances. Un autre subterfuge serait de réduire la durée du mandat du président de la Cour Suprême et ses pouvoirs discrétionnaires. Nawaz Sharif n'est peut-être pas aussi opposé qu'il y paraît à circonscrire dans l'absolu l'activisme du président de la Cour Suprême si on se souvient qu'en 1997, alors premier ministre, il avait eu maille à partir avec le plus haut magistrat de l'état, Sajjad Ali Shah.

La nomination au poste de gouverneur du Pendjab de Salman Tasser, un ancien compagnon de route du PPP et, il y a peu, ministre du gouvernement intérimaire, estle signe d'une volonté de ne pas abandonner cette province cruciale à la PML-N. Si la PML-N devait décider de passer d'un soutien au coup par coup au gouvernement sans participation ministérielle à une opposition plus résolue au gouvernement, le PPP pourrait être tentée d'attirer à lui des députés de la PML-Q. Les tergiversations du gouvernement actuel sur le rétablissement des juges démis - symbole de la lutte contre l'autocratie pour l'opinion publique - et sur une opposition résolue à Musharraf, ont un coût élevé en terme d'image, notamment au profit de la PML-N, mais il pourrait être amorti si l'objectif du retour à une démocratie parlementaire n'est pas perdu de vue. Il faut garder à l'esprit, que le PPP, bien qu'il soit le premier parti du pays, n'a gouverné que cinq ans sur les trente dernières années et entend rester au pouvoir sur l'ensemble de la législature. Une gouvernance qui s'annonce complexe au regard de l'ampleur des problèmes auxquels le pays est confronté.

\section{Des défis de taille à relever}

21 Parmi les mesures annoncées pour établir les intentions démocratiques du nouveau gouvernement, Gilani, dans son discours d'investiture, avait annoncé la libération des juges encore en résidence surveillée, la levée prochaine des entraves à la liberté des médias, ainsi que de l'interdiction des syndicats étudiants et ouvriers ${ }^{14} . \mathrm{Il}$ avaitaussi rappelé que la tâche première de son gouvernement serait le rétablissement d'un climat de concorde sans sacrifier à la lutte contre le terrorisme, s'inscrivant dans le droit fil de la campagne électorale de Benazir Bhutto qui avait même évoqué un renforcement de la fort décriée coopération avec les Etats-Unis. Le nombre d'attentats-suicide est passé de 6 en 2006 à 62 en 2007.

22 Face à la talibanisation des FATA, le gouvernement a promis des réformes politiques et une intégration socio-économique facilitée en partie par la création de Reconstruction Opportunities Zones, notamment financées par les Américains. Il est question d'étendre à ces zones tribales, administrées à partir d'Islamabad en vertu de l'article 247 de la constitution, le " Political Parties Act », ce qui permettrait aux partis politiques d'y opérer officiellement. Par ailleurs, les Frontier Crime Regulations, une juridiction pénale locale promulguée en 1901 et visantalors à préserver les intérêts géostratégiques des Britanniques dans la région sans altérer les coutumes locales, sont appelées à disparaitre. 
Mais pour les militants talibans et des chefs tribaux, cela serait l'occasion de leur substituer la loi islamique (sharia). Il y a le précédent de l'accord en 16 points signé le 21 mai dans la région de Malakand entre le gouvernement provincial dirigé par l'ANP et la milice de Maulana Fazlullah dont l'activisme armé dans la vallée de Swat avait amené au déploiement de 20000 hommes de troupes au cours de l'automne dernier. Les miliciens du Tehreek-e-Nifaz-e-Shariat-e-Muhammadi (TNSM, Mouvement pour l'application des lois islamiques) s'engagent à renoncer à l'usage des armes contre les organes de l'Etat en échange de quoi ils bénéficient d'une amnistie et d'une certaine latitude pour diffuser leur code de conduite sociétal. Ce qui pour certains ressemble à une politique d'apaisement vis-à-vis d'éléments extrémistes survient alors que, à l'inverse de 2002 où le MMA avait remporté tous les sièges dans la vallée de Swat, sept des neuf sièges en jeu au niveau provincial et national sont revenus cette fois-ci à l'ANP (le deuxième siège national fut remporté par le PPP) ${ }^{15}$.

La possibilité de voir ce type d'accord se répliquer dans les FATA frontalières de l'Afghanistan n'est pas à exclure. Alors que par ailleurs des boutiques de CDs/DVDs et des écoles de filles continuent à être la cible d'attaques, des pourparlers sont en cours entre des représentants du gouvernement fédéral, l'armée, les dirigeants tribaux et le Tehrik-iTaliban Pakistan (TTP) qui, depuis décembre 2007, fédère, sous la houlette, de Baitullah Mehsud, différents groupes militants tribaux opérant dans les 7 districts des FATA et dans les 24 districts des PATA (Provincially Administered Tribal Areas). Les Américains suivent avec beaucoup d'attention ces discussions en redoutant qu'un compromis conduise à des libérations de militants et à un repositionnement de l'armée pakistanaise qui en fait masquerait une retraite et l'abandon de territoires aux talibans, facilitant aussi les opérations d'Al-Qaeda. Les précédents accords de paix avaient permis aux militants de se remettre en ordre de bataille. Baitullah Mehsud, qui opère au Sud-Waziristan, a d'ores et déjà déclaré qu'un accord ne signifierait pas un renoncement à la poursuite du jihad en Afghanistan. En tirant le 15 mai un missile à partir d'un drone contre le village de Damadola (agence de Bajaur), déjà visé en janvier 2006, et, plus récemment, en décidant $\mathrm{du}$ bombardement d'un poste frontalier dans l'agence de Mohmand qui coûta la vie à 11 membres des paramilitaires des Frontier Corps le 10 juin, les Américains firent entendre qu'ils n'entendaient pas baisser la garde et attendaient de l'arméepakistanaise qu'elle ne faillisse dans la mission pour laquelle elle avait reçu une part importante des onze milliards de dollars accordés au Pakistan depuis 2001. La réponse du côté des talibans ne s'était pas faite attendre avec le premier attentat suicide à Mardan contre des forces de sécurité depuis la prise de fonctions du nouveau premier ministre. Les visites régulières de hauts-fonctionnaires américains visant à s'assurer que le Pakistan ne faiblirait pas dans la lutte contre le terrorisme ne sont guère goûtées par l'opinion publique. La nécessité de redéfinir les liens avec les Etats-Unis qui ne ferait pas du Pakistan un Etat mercenaire est souvent soulignée. Mais Washington dispose toutefois d'importants moyens de pression pour jouer d'influence au niveau bilatéral et multilatéral. Plus de dix milliards de dollars sont allés au Pakistan depuis 2001 et l'actuelle délicate passe financière ne risque pas d'alléger cette dépendance. Il fut rapporté que le Pakistan avait demandé aux Etats-Unis l'envoi de 500000 tonnes de blé dans le cadre de la loi publique-480 (PL-480) traitant d'assistance alimentaire ${ }^{16}$.

Il sera intéressant de voir si l'espace de négociations offert aux talibans pakistanais est étendu aux groupes nationalistes baloutches. De manière générale, le nouveau gouvernement a promis d'agir en faveur de plus d'autonomie régionale. Il y l'engagement 
symbolique à rebaptiser la NWFP en Pakhtunkhwa. L'intégration des FATA dans cette province n'est toutefois pas à l'ordre du jour car cela pourrait alimenter un irrédentisme pachtoune dont se méfient notamment ceux qui à Islamabad veulent garder la haute main sur une zone-clé pour la conduite des intérêts stratégiques du Pakistan en Afghanistan. Le premier ministre a promis de supprimer d'ici un an la «liste convergente » qui permet à la législation fédérale de primer sur la législation provinciale dans des domaines qui pourraient être pourtant du ressort exclusif de celle-ci. Des initiatives ont été prises pour aplanir les tensions au Baloutchistan. Le PPP a officiellement présenté ses excuses au peuple baloutche pour les atrocités et les injustices commises par le passé (personne n'a oublié que Zulfikar Ali Bhutto avait en son temps décidé d'opérations militaires massives) et annoncé la création d'une commission pour la vérité et la réconciliation. Akhtar Mengal, ancien ministre en chef de la province et leader du BNP, qui était emprisonné depuis septembre 2006 pour actions de nature terroriste, a été libéré. Il faudra toutefois des mesures bien plus concrètes, comme la démilitarisation de la province, la réhabilitation des personnes déplacées, des informations sur les personnes disparues, ou encore un partage plus équitable des ressources pour combler le déficit de confiance qui existe entre les Baloutches et l'establishment militaro-civil à Islamabad.

Aux défis intérieurs de nature extrémiste ou nationaliste, le gouvernement a pris ses fonctions dans un contexte économique particulièrement défavorable, conjonction de facteurs exogènes et endogènes. Le budget présenté le 11 juin a déjà revu à la baisse la croissance pour l'année 2007-2008 en la chiffrant à 5,8\%. L'escalade des prix du pétrole a obligé à plusieurs ajustements locaux, ce qui a accéléré un mouvement inflationniste particulièrement sensible sur les produits de première nécessité. Si officiellement le taux d'inflation est cette année de l'ordre de $11 \%$ (contre 7,8\% en 2006-2007), le prix des aliments de base aurait augmenté de plus de $30 \%$ en un an. Le Programme Alimentaire Mondial annonça en avril 2008 que près de la moitié de la population pakistanaise étaient dorénavant menacée d'insécurité alimentaire. Dans le même temps, le pays est confronté à un déficit sans précédent dans la production d'électricité faute d'anticipation de la hausse rapide de la demande. Outre la promesse d'une société moderne et tolérante derrière le slogan de la "modération éclairée ", Musharraf, comme en son temps Ayub Khan, a essayé de compenser le manque démocratique, par une exceptionnelle croissance économique. Il apparaît que celle-ci était en trompe l'oeil. L'explosion de la vente de téléphones mobiles ne peut dissimuler le fait que la croissance a surtout permis de satisfaire les appétits de consommation d'une minorité. Le taux d'épargne est tombé à $14 \%$ du PIB et les investissements étrangers sont surtout allés ces dernières années vers des secteurs lucratifs comme les communications, les placements financiers, l'immobilier et l'exploration pétrolière et gazière, mais sans réellement renforcer la structure industrielle du pays afin de créer les emplois et générer des revenus à l'exportation si nécessaires. Le déficit de la balance commerciale a atteint un niveau record s'accompagnant d'une baisse des réserves de change alors que l'importance d'un déficit budgétaire (7\%) générateur d'inflation laisse peu de marge de manoeuvre au gouvernement sans accepter de passer sous les fourches caudines de créanciers internationaux qui demandent une remise en cause de diverses subventions publiques pour les produits pétroliers, l'éléctricité ou encore le blé.

Près de quatre mois a près des élections qui furent saluées comme un renouveau démocratique, mettant un terme à la troisième période de gouvernance militaire, il reste bien difficile de définir les contours des nouveaux équilibres institutionnels et de 
déterminer la nature précise des compromis à venir et des mécanismes mis en place pour régler les rivalités entre les différentes sources de pouvoir. L'absence de cohésion au sein de la coalition au pouvoir alimente l'indécision. La population, qui espèrait que les intérêts personnels seraient oubliés au moins le temps de renforcer les institutions démocratiques, s'inquiète d'une inaction prolongée et de l'absence de perspectives dans un contexte qui appele pourtant à des mesures de redressement rapides. Une consolation réside cependant dans le fait que les nouveaux médias indépendants et la société civile veulent peser sur le jeu politique et veiller à ce qu'il n'y ait pas de retour en arrière.

\section{NOTES}

1. Quaid-e-Azam ("Grand Leader") fait référence au "Père de la Nation", Mohammad Ali Jinnah. The News, 24 février 2008.

2. Etude réalisée par le Free and Fair Election Network (FAFEN). Dawn , 8 décembre 2007.

3. L'assemblée nationale est composée de 272 «sièges généraux " déterminés lors d'un scrutin à un tour auxquels s'ajoutent 60 sièges réservés aux femmes et 10 sièges réservés aux minorités attribués en proportion des scores réalisés par les différents partis. 6 sièges devront encore être attribués lors d'élections partielles de la fin juin.

4. Les deux-tiers des sièges remportés par la PML-N le furent dans seulement 12 districts du nord et de l'est du Pendjab (il y a au total 110 districts au Pakistan). Autre constatation, la PML-N semble avoir bénéficié d'un report conséquent des voix du JI vers ses candidats. Mais la concentration géographique du vote PML-N se traduit aussi par le fait qu'il obtint moins de votes au niveau national que la PML-Q (20\% contre $23 \%$ pour ce dernier).

5. Ainsi Rao Sikandar Iqbal, qui était ministre de la défense, fut nettement battu dans la circonscription d'Okara au Pendjab. Preuve s'il en faut de la versatilité des personnalités politiques, Iqbal avait remporté ce même siège en 2002 comme candidat du PPP avant de passer dans la majorité d'alors. Un autre transfuge vainqueur en 2002 sur une candidature PPP (district de Jhang, Pendjab), Faisal Saleh Hayat, qui fut lui aussi récompensé par un ministère, fut par contre réélu sous l'étiquette PML-Q.

6. Kaiser Bengali \& Zaheer Gazdar, 'Anatomy of elections', Dawn, 6 April 2008. Pour des analyses des tendances électorales, voir aussi Haris Gazdar, 'The health of the federation', Dawn, 13 février 2008; Herald, vol. 39, n 3, March 2008; Kaiser Bengali \& Zaheer Gazdar, 'Anatomy of elections', Dawn, 6 April 2008.

7. Rappelons ici que Nawaz Sharif pouvait avoir quelques griefs personnels à l'encontre de Musharraf qui l'avait forcé à sept années d'exil et avait provoqué en 2001 une scission au sein de son parti, la PML, pour faire de la PML-Q un instrument à son service.

8. Fazlur Rehman a même été battu dans le fief historique du JUI-F, la circonscription de Dera Ismail Khan, dont son père avait remporté le siège en 1970. Il ne dut son salut qu'à une victoire étriquée à Bannu. Sur l'action du MMA au pouvoir, voir : Nazish Brohi, The MMA Offensive: Three Years in Power, 2002-2005, Islamabad : ActionAid Pakistan, 2006.

9. Un seul député de la PML-Q a décidé de rester dans l'opposition pour cause de querelle de clan. Ce large ralliement ne fut pas sans contrepartie puisque 44 des 62 élus de cette majorité composite ont reçu un portefeuille au sein du gouvernement. 
10. Un exemple, le jour de la prestation de serment, les ministres de la PML-N portèrent un brassard noir, et préférèrent rester debout de longues minutes en attendant le président afin de ne pas avoir à se lever à son arrivée.

11. Dawn, 14 février 2008.

12. Voir: Ayesha Siddiqa, Military Inc.: InsidePakistan's Military Economy, Karachi: Oxford University Press, 2007.

13. Cet article avait été inscrit dans la Constitution sous Ziaul Haq, en 1985, supprimé en 1997 lorsque Nawaz Sharif était premier ministre, et réintroduit par Musharraf en 2003.

14. Le syndicalisme au niveau étudiant n'a jamais totalement disparu, même si cela fut de façon officieuse. Le plus organisé est certainement l'Islami-Jamiat-e Tuleba (IJT), le syndicat étudiant $\mathrm{du}$ JI, notamment très actif sur le campus à l'université du Pendjab à Lahore, et au sein duquel des dirigeants politiques de tout bord ont fait leurs classes. Le MQM était né du All Pakistan Mohajir Students Organisation.

15. Parmi les engagements des troupes du Maulana Fazlullah, celui de ne plus s'attaquer aux forces de sécurité et aux installations du gouvernement, de livrer les éventuels combattants étrangers, mais aussi de ne pas s'attaquer aux barbiers, de ne pas s'opposer à l'éducation des filles et aux campagnes d'immunisation. Quant à la radio du maulana, elle pourra continuer à émettre et la madrasa de Fazlullah dans le village de Imam Dheri sera convertie en université islamique sous administration conjointe gouvernement/taliban. Quelques semaines plus tôt, le beau-père de Fazlullah, Sufi Mohammed, avait été libéré de prison contre la promesse d'un renoncement à la lutte armée pour imposer la sharia et d'un retrait progressif de l'armée. Sufi Mohammed, fondateur du TNSM, avait été incarcéré à son retour d'Afghanistan où il avait entraîné quelque dix mille militants de son parti pour combattre les Américains en 2001. Nombre d'entre eux avaient été tués.

16. Dawn, 15 juin 2008.

\section{RÉSUMÉS}

Le 18 février 2008, les électeurs pakistanais infligèrent un camouflet au régime du président Musharraf en votant majoritairement pour les partis qui, entre deux gouvernances militaires, s'étaient succédés au pouvoir dans les années quatre-vingt dix. En réponse au verdict populaire, le Pakistan Peoples Party, le parti des Bhutto-Zardari, et la Pakistan Muslim Leaguede Nawaz Sharif décidérent de joindre leurs forces avec l'ambition déclarée de rétablir la suprématie du parlement et l'indépendance du pouvoir judiciaire. Mais la coalition gouvernementale doit non seulement gérer ses différends mais aussi accommoder l'esprit des réformes aux positions des autres lieux de pouvoir. Pendant ce temps, la population cherche des raisons d'espérer dans un contexte où la violence ne recule pas et où l'économie languit.

\section{INDEX}

Mots-clés : constitution, démocratie, élection, Musharraf, Pakistan, parti politique, taliban 


\section{AUTEUR}

\section{GILLES BOQUÉRAT}

Gilles Boquérat est chercheur résident à l'Institut d'Etudes Stratégiques, Islamabad et chercheur associé au Centre d'Etudes de l'Inde et de l'Asie du Sud, Paris. 